\title{
El desempeño en los cursos cuantitativos como predictor de éxito en los estudios universitarios
}

\author{
José Vega Vilca | jose.vega23@upr.edu \\ Universidad de Puerto Rico, Recinto de Río Piedras \\ Wanda Velázquez Rosado | wanda.velazquez@upr.edu \\ Universidad de Puerto Rico, Recinto de Río Piedras \\ Wanda Villafañe Cepeda | wanda.villafane1@upr.edu \\ Universidad de Puerto Rico, Recinto de Río Piedras
}

Recibido: 15 de agosto de 2016

Aceptado: 27 de septiembre de 2016

\section{RESUMEN}

Este estudio investiga las variables que pueden predecir el éxito universitario. Se utilizó una muestra de 202 alumnos que culminaron estudios en diciembre de 2013 y mayo de 2014. Se estudiaron las variables aprovechamiento y aptitud en matemática, género del estudiante, tipo de escuela, índice general de solicitud, índice de los cursos cuantitativos, promedio de grado y tiempo de estudio. Para analizar los datos se usó el partial least squares path modeling y el análisis de regresión logística. Se encontró que el desempeño de los estudiantes en los cursos cuantitativos es un buen predictor del éxito en sus estudios.

Palabras clave: educación superior, administración de empresas, cursos cuantitativos, aprovechamiento matemático, éxito estudiantil

\section{ABSTRACT}

This study investigates the variables that can predict university success. A sample of 202 students who completed studies in December 2013 and May 2014 was used. The variables achievement and aptitude in mathematics, student gender, type of school, general index of application, quantitative courses index, grade point average, and study time were studied. The data were analyzed using the partial least squares path modeling and logistic regression analysis. The results revealed that the performance of students in the quantitative courses is a good predictor of success in their studies.

Keywords: higher education, business administration, quantitative courses, math achievement, student success 
Cada día es más importante que las universidades que preparan estudiantes de administración de empresas requieran que sus ingresados y egresados tengan competencias cuantitativas (Gordon, 2010). Es necesario que los alumnos tengan dominio de las destrezas cuantitativas para que puedan resolver problemas y tomar decisiones efectivas, tanto en sus estudios universitarios como en su desempeño laboral.

A pesar de que la mayoría de los programas subgraduados requieren la aprobación de cursos cuantitativos, la tasa de estudiantes que aprueban estos cursos es muy baja (Gupta, Harris, Carrier, \& Caron, 2006; Larrazolo, Backhoff, \& Tirado, 2013). Particularmente en la Universidad de Puerto Rico, Recinto de Río Piedras (UPRRP), se ha encontrado que existe un alto por ciento de fracasos de los estudiantes en los cursos cuantitativos, como lo son Precálculo y Cálculo.

El porcentaje de fracasos en el curso Métodos Cuantitativos para Administración de Empresas I (MECU 3031) que ofrece la Facultad de Administración de Empresas (FAE), de la UPRRP, durante los años académicos 2002-2003 al 2014-2015, se mantuvo en alrededor de un $50 \%$ durante el primer semestre y un 53\% durante el segundo semestre. Este alto porcentaje de fracaso incide en que muchos de los estudiantes fracasen en cursos subsiguientes y, por ende, no pueden tener éxito en la culminación de su carrera universitaria en el tiempo esperado. Precisamente, el objetivo principal de este estudio es investigar las variables que pueden predecir el éxito en los estudios universitarios de los estudiantes en la FAE.

\section{Revisión de literatura}

Las universidades quisieran determinar parámetros para reconocer las destrezas que poseen los estudiantes de nuevo ingreso y cómo estas inciden en el éxito en sus estudios universitarios (Parker, 2005). Generalmente, el éxito universitario está asociado con las calificaciones que obtienen los estudiantes y el tiempo que les toma completar su grado. Investigaciones realizadas apuntan a que existen varios factores que inciden en el éxito en los estudios uni- 
versitarios, tales como: aprovechamiento y aptitud en matemática, promedio de escuela superior, género del estudiante, escuela de procedencia, ingreso familiar, índice general de solicitud (IGS), calificaciones en cursos cuantitativos, promedio de grado, tiempo de estudio y factores socioeconómicos.

\section{Investigaciones que incluyen las variables de aprovechamiento ma- temático e IGS}

Parker (2005) realizó una investigación cuyo propósito principal fue explorar la relación entre la retención estudiantil en la Universidad de Clarion y el éxito de los alumnos en las matemáticas. Los hallazgos demostraron que los estudiantes que tenían más éxito en los cursos de matemáticas tenían mayor probabilidad de permanecer en la universidad y graduarse en cuatro años; esto implica que las matemáticas facilitan el éxito de los estudiantes en su carrera académica.

Lesik (2007) realizó un estudio en el cual participaron 1,276 estudiantes de nuevo ingreso de una universidad, que estudiaban a tiempo completo entre los años 2000 y 2002. Estos estaban matriculados en el curso de álgebra intermedia (matemática básica) o pasaron directamente a tomar los cursos de matemática del nivel universitario. Se encontró que aquellos que participaron en los cursos de matemática básica se mantuvieron más tiempo matriculados en sus estudios universitarios; es decir, que el riesgo de que aquellos estudiantes que tomaron los cursos de matemática básica abandonaran la universidad fue significativamente menor, al compararlos con aquellos que no los tomaron.

Vega Vilca y Agosto (2010) realizaron un estudio para predecir el aprovechamiento académico de los egresados del programa subgraduado de la FAE. Los autores definieron el concepto de "egresado de éxito académico" como un estudiante con promedio de graduación de al menos 3.33, que logró terminar su bachillerato en un promedio de cinco años o menos. En el estudio participaron 391 egresados subgraduados que culminaron estudios en diciembre de 2008, mayo de 2009 y verano de 2009. Los investigadores encontraron que el género del estudiante, así como el tipo de es- 
cuela superior de procedencia (pública o privada) no son buenos predictores del éxito académico; sin embargo, el IGS constituye un buen predictor de éxito. Contrario a las conclusiones de Matos Díaz (2008), en este trabajo se concluye que el IGS puede ser un buen predictor del éxito del egresado, siempre que se tenga valores suficientemente altos de este índice. Cuando se comparan dos grupos de estudiantes con IGS diferenciado por 25 puntos, la posibilidad de encontrar un egresado de éxito es mayor en el grupo con IGS más alto (2.67 veces). También encontraron que el índice de los cursos de métodos cuantitativos (Imecu) es un índice nuevo, propuesto en este trabajo, que predice el éxito del futuro egresado. Cuando se comparan dos grupos de estudiantes con Imecu diferenciado por un punto, la posibilidad de encontrar un egresado de éxito es mayor en el grupo con Imecu más alto (3.09 veces).

\section{Investigaciones que incluyen variables socioeconómicas}

DeNicco, Harrington y Fogg (2015) estudiaron los factores de retención de estudiantes de primer año de un colegio comunitario. Estos consideraron los factores: género, raza, características de la escuela superior, puntaje en el examen de entrada, desempeño durante el primer año y cursos remediales durante el primer año. Los resultados indican que el desempeño en el primer año es el predictor más fuerte en la retención. El grade point average (GPA) y el número de créditos obtenidos en primer año son predictores estadísticamente significativos en la retención del estudiante. Se encontró que todas las demás variables no fueron significativas para explicar la retención de los estudiantes.

Coronado Ramírez y Sandoval Bravo (2012) realizaron una investigación para determinar si el género, los resultados de la sección de matemáticas de la prueba de aptitud académica y algunos factores demográficos (estado civil, situación laboral y tipo de institución de procedencia) inciden en el rendimiento en los cursos de matemáticas de los estudiantes de nuevo ingreso de la Universidad de Guadalajara, México. Los investigadores no encontraron variables que pudieran establecer una correlación en su rendimiento. Ellos concluyeron que existe otro tipo de variables socioeconómi- 
cas o sociohistóricas que pudieran permitir determinar el rendimiento en matemáticas de los estudiantes.

Arias Ortiz y Dehon (2013) realizaron una investigación en la cual analizaron los factores que influenciaban en el abandono de los estudios universitarios por parte de los alumnos de la Université Libre de Bruxelles. Se encontró que a pesar de que muchos comenzaban sus estudios, muy pocos completaban sus grados. También descubrieron que los alumnos provenientes de clase socioeconómica baja eran más vulnerables a abandonar sus estudios y los factores económicos les impedían matricularse nuevamente. Por otro lado, encontraron que una proporción alta de alumnos que volvieron a estudiar en la Universidad, pero en un campo de estudio distinto al que estaban antes de abandonarla, culminaron sus estudios, en comparación a los que se matricularon nuevamente en el mismo campo de estudio en el que estaban originalmente. Indican que esto sugiere que las universidades deben reevaluar los mecanismos disponibles para trabajar con los fracasos y guiar a los estudiantes en la selección que estos realicen de las profesiones que desean estudiar.

Vázquez Calle y Cabrera Pérez (2004) realizaron un estudio cuyo objetivo principal fue identificar y evaluar la importancia relativa de las características académicas y no académicas, que explican el éxito o fracaso en completar el grado de bachillerato de los estudiantes de nuevo ingreso, de la Universidad de Puerto Rico en Cayey (UPRC) de los años académicos 1995 al 1998. Específicamente consideraron las siguientes variables independientes para estimar el modelo de regresión logística, mediante el cual se midió el efecto neto de cada predictor sobre la probabilidad de éxito en graduarse: variables académicas preuniversitarias (aptitud en matemática, aptitud verbal, aprovechamiento en matemáticas, español e inglés, índice académico de escuela superior); variables académicas universitarias (índice académico obtenido en su primer año de estudios, cumplimiento con el índice mínimo de 2.00 y el área académica de admisión); variables no académicas (género, escuela superior de procedencia, ingreso del hogar, educación del padre, educación de la madre y generación del estudiante). 
Con relación a las variables académicas universitarias, los investigadores indicaron que de todos los estudiantes que obtuvieron un promedio académico menor de 2.00 en el primer año de estudios en la UPRC, el $88.7 \%$ no completó el bachillerato. Adicional a esto, los resultados indican que el desempeño académico de los estudiantes al finalizar el primer año de universidad determina significativamente el éxito o fracaso posterior en completar el grado académico.

Relacionado con el área académica en las que estaban matriculados los estudiantes de nuevo ingreso que participaron en la investigación, encontraron que las áreas de Ciencias Naturales y Administración de Empresas reflejaron una tasa de éxito en graduarse mayor, que los estudiantes de las áreas de Ciencias Sociales y Humanidades.

En relación con las variables no académicas, encontraron que las mujeres tuvieron una tasa ligeramente mayor de éxito en graduarse que los hombres. Además, no encontraron evidencia de que el tipo de escuela superior de procedencia tuviera un efecto significativo en la probabilidad de completar o no el grado de bachillerato. En particular, los investigadores encontraron que los estudiantes de escuela privada reflejaron una probabilidad de éxito tan solo $5 \%$ mayor que sus homólogos de escuela pública.

\section{Metodología}

La metodología utilizada en este estudio consistió en la recopilación y generación de una base de datos y el posterior análisis estadístico de los mismos usando las técnicas estadísticas partial least squares path modeling (PLSPM) y análisis de regresión logística (RL).

La base de datos utilizada fue proporcionada por la Oficina de Planificación Estratégica y Presupuesto de la UPRRP, y consta de la información de los egresados subgraduados de la FAE que culminaron estudios en diciembre de 2013 y mayo de 2014. Se analizaron los datos de 202 egresados cuya primera concentración fue Contabilidad (106), Economía (1), Finanzas (17), Gerencia de Mercadeo (40), Gerencia de Operaciones y Suministros (10), Gerencia 
de Recursos Humanos (19) y Sistemas Computadorizados de Información (9). Se excluyeron a los estudiantes de la concentración de Administración de Sistemas de Oficina, ya que ellos no toman los cursos de naturaleza cuantitativa considerados en este estudio. También se excluyeron a los estudiantes de otras concentraciones, porque presentaban datos incompletos.

Se analizaron las variables: aprovechamiento y aptitud en matemática en la Prueba de Evaluación y Admisión Universitaria, género del estudiante, tipo de escuela (pública o privada), IGS, índice de notas de los cursos cuantitativos, promedio de graduación y tiempo de estudio (en años). Para realizar el análisis de los datos se consideraron las notas de los estudiantes en cursos medulares de naturaleza cuantitativa que ofrece la FAE; específicamente, se analizaron las notas de los siguientes cursos: Métodos Cuantitativos para Administración de Empresas I y II (MECU 3031), Métodos Cuantitativos para Administración de Empresas II (MECU 3032), Estadística para Administración de Empresas I (ESTA 3041), Estadística para Administración de Empresas II (ESTA 3042), Introducción a la Estadística para la Administración de Empresas (ESTA 3045), Introducción a los Fundamentos de Contabilidad I (CONT 3105), Introducción a los Fundamentos de Contabilidad II (CONT 3106), Mercados e Instituciones Financieras (FINA 3107), Gerencia Financiera (FINA 3106), Introducción a la Gerencia de Operaciones y Suministros (GEOP 4315) y Gerencia Estratégica (ADMI 4007).

Para un mejor análisis del desempeño de los estudiantes en los cursos cuantitativos analizados, se transformaron las notas obtenidas en índices de notas de los cursos. Estos índices valoran la ejecutoria del estudiante en cada uno de los cursos analizados y para ser calculado fue necesario asignar puntaje a los diferentes calificativos, así: $\mathrm{A}=4, \mathrm{~B}=3, \mathrm{C}=2, \mathrm{D}=1$ y $\mathrm{F}=0$. Si un estudiante aprobó el curso en la tercera oportunidad con calificativo B, pero en las dos veces anteriores obtuvo $\mathrm{F}$ y $\mathrm{D}$, su índice fue $(0+1+3) / 3=1.33$.

En el estudio participaron 118 estudiantes del género femenino y 84 estudiantes del género masculino. Del total de estudiantes, 135 provenían de escuelas privadas y 67 de escuelas públicas, como lo ilustra la Tabla 1. 
Tabla 1

\begin{tabular}{lc} 
Datos cualitativos & \\
\hline Variable & Frecuencias \\
\hline Género del estudiante & Femenino $=118(58.4 \%)$ \\
& Masculino $=84(41.6 \%)$ \\
Tipo de escuela superior & Privada $=135(66.8 \%)$ \\
& Pública $=67(33.2 \%)$ \\
\hline
\end{tabular}

Fuente: Elaboración propia.

La Tabla 2 presenta una descripción de los datos cuantitativos para cada una de las variables estudiadas. Los estadísticos calculados fueron: valor mínimo (Mín.), primer cuartil (Q1), mediana, promedio, tercer cuartil (Q3) y valor máximo (Máx.).

Tabla 2

Datos cuantitativos

\begin{tabular}{lcccccc}
\hline Variable & Mín. & Q1 & $\begin{array}{c}\text { Media- } \\
\text { na }\end{array}$ & Promedio & Q3 & Máx. \\
\hline Tiempo de estudio (años) & 4.0 & 5.0 & 5.0 & 5.5 & 6.0 & 20.0 \\
Índice general de solicitud (IGS) & 187.0 & 300.0 & 316.0 & 315.5 & 333.0 & 362.0 \\
Aprovechamiento matemático & 413.0 & 579.2 & 629.0 & 620.6 & 671.0 & 788.0 \\
Aptitud matemática & 382.0 & 578.5 & 638.0 & 631.8 & 695.2 & 766.0 \\
Promedio de graduación & 2.55 & 3.04 & 3.29 & 3.30 & 3.56 & 4.00 \\
Índice de MECU 3031 & 0.20 & 1.50 & 2.00 & 2.30 & 3.00 & 4.00 \\
Índice de MECU 3032 & 0.50 & 2.00 & 2.00 & 2.38 & 3.00 & 4.00 \\
Índice de CONT 3105 & 0.50 & 2.00 & 3.00 & 2.89 & 4.00 & 4.00 \\
Índice de CONT 3106 & 0.50 & 3.00 & 3.00 & 3.02 & 4.00 & 4.00 \\
Índice de ESTA 3041-3042-3045 & 0.50 & 2.00 & 2.75 & 2.71 & 3.50 & 4.00 \\
Índice de ADMI 4007 & 1.00 & 3.00 & 4.00 & 3.43 & 4.00 & 4.00 \\
Índice de GEOP 4315 & 1.00 & 2.00 & 3.00 & 2.90 & 4.00 & 4.00 \\
Índice de FINA 3106 & 0.30 & 3.00 & 3.00 & 3.18 & 4.00 & 4.00 \\
Índice de FINA 3107 & 0.30 & 2.12 & 3.00 & 2.99 & 4.00 & 4.00 \\
\hline
\end{tabular}

Fuente: Elaboración propia. 


\section{Análisis de los datos y resultados}

La base de datos fue analizada usando dos técnicas estadísticas; estas son:

1. PLSPM: Después de hacer grupos de variables que se relacionan entre sí, este análisis determina la existencia de relaciones entre los bloques de variables, también llamados constructos o variables latentes. Se estudió la relación entre cuatro constructos y se cuantificaron las conexiones del modelo propuesto, el cual define el desempeño general del egresado de la FAE; esto es, desempeño en la escuela superior, los cursos introductorios, los cursos intermedios y avanzados, y general (éxito).

2. RL: Determina la relación funcional entre una variable dependiente cualitativa (dos o más categorías) y una o más variables explicativas independientes o covariables, ya sean cualitativas o cuantitativas. En este trabajo se estudió la relación entre la variable dependiente éxito académico del estudiante, que se definió como aquel estudiante que finalizó su bachillerato en cinco años o menos con promedio de graduación de, por lo menos, tres puntos; y las variables independientes: género del estudiante, escuela de procedencia, IGS y los índices de los cursos cuantitativos en estudio. Los conceptos de odds y odds ratio (OR) son muy importantes para comparar la ejecutoria estudiantil en dos categorías de las variables del estudio.

\section{PLSPM}

Se agruparon las variables que se relacionan entre sí con la finalidad de generar constructos, los cuales se visualizan como nuevas variables que no son observables directamente, sino a partir de las variables observables asociadas a dicho constructo. Se estudió la relación entre cuatro constructos que relacionan el desempeño del estudiante egresado de la FAE. La Tabla 3 muestra los constructos y las variables observadas asociadas a cada uno de ellos. 
Tabla 3

Constructos y sus variables asociadas del modelo en estudio

\begin{tabular}{lc}
\hline Constructo C1: Desempeño en la escuela superior \\
\hline IGS & Índice General de Solicitud \\
APRM & Aprovechamiento Matemático \\
APTM & Aptitud Matemática \\
\hline Constructo C2: Desempeño en los cursos introductorios \\
\hline CONT 3105 & Introducción a los Fundamentos de Contabilidad I \\
CONT 3106 & Introducción a los Fundamentos de Contabilidad II \\
MECU 3031 & Métodos Cuantitativos para Administración de Empresas I \\
MECU 3032 & Métodos Cuantitativos para Administración de Empresas II \\
ESTA & Estadística: ESTA 3041, ESTA 3042, ESTA 3045 \\
\hline Constructo C3: Desempeño en los cursos intermedios y avanzados \\
\hline FINA 3106 & Gerencia Financiera \\
FINA 3107 & Mercados e Instituciones Financieras \\
ADMI 4007 & Gerencia Estratégica \\
GEOP 4315 & Introducción a la Gerencia de Operaciones y Suministros \\
\hline Constructo C4: Desempeño general (éxito) \\
PRGR
\end{tabular}

Fuente: Elaboración propia.

El modelo que se utiliza en el estudio es un sistema de relaciones lineales entre cuatro bloques de variables (constructos), tal como ilustra la Figura 1. Estas relaciones lineales involucran cuatro hipótesis sobre desempeño de los estudiantes de la FAE:

H1: C1 $\rightarrow$ C2, el desempeño en la escuela superior influye positivamente en el desempeño de los cursos introductorios universitarios.

H2: C2 $\rightarrow$ C3, el desempeño en los cursos introductorios universitarios influye positivamente en el desempeño de los cursos intermedios y avanzados universitarios.

H3: C2 $\rightarrow$ CA, el desempeño en los cursos introductorios universitarios influye positivamente en el desempeño general (éxito)

H4: C3 $\rightarrow$ C4, el desempeño en los cursos intermedios y avanzados universitarios influye positivamente en el desempeño general (éxito) 


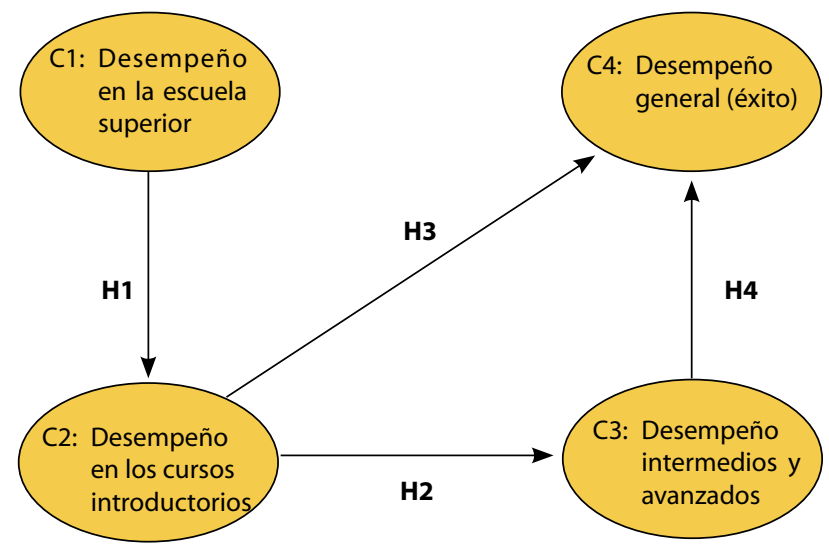

Figura 1. Diagrama del modelo en estudio. Fuente: Elaboración propia.

La metodología PLSPM trabaja básicamente en tres pasos. Primero, se calculan ponderaciones (weights) en cada constructo, para combinarse linealmente con las variables asociadas a cada constructo y originar valores (scores) representativos. Segundo, se calculan las correlaciones (loadings) entre los scores y las variables asociadas a cada constructo. Tercero, se calculan los coeficientes del sistema de relaciones lineales de la Figura 1. La Tabla 4 presenta los cálculos mencionados en los dos primeros pasos, además presenta el cálculo de communality para cada variable asociada a su constructo. Se observa que el valor más bajo es para la variable ADMI 4007, la cual aporta solo el $15.3 \%$ de su variabilidad al constructo C3. Esta variabilidad es mucho menor que el $45 \%$ que sugieren los autores de este trabajo, a pesar de que Sánchez (2013) sugiere una variabilidad de $50 \%$. Este resultado motivó el retiro de dicha variable y el reprocesamiento de los datos, con una variable menos asociada al constructo C3. 
Tabla 4

Ponderaciones de las variables de cada constructo

\begin{tabular}{lcccc}
\hline Variable & Constructo & Weight & Loading & Communality \\
\hline IGS & C1 & 0.389 & 0.821 & 0.675 \\
APRM & C1 & 0.425 & 0.891 & 0.794 \\
APTM & C1 & 0.337 & 0.896 & 0.802 \\
\hline MECU 3031 & C2 & 0.287 & 0.713 & 0.508 \\
MECU 3032 & C2 & 0.262 & 0.679 & 0.461 \\
CONT 3105 & C2 & 0.258 & 0.684 & 0.468 \\
CONT 3106 & C2 & 0.285 & 0.718 & 0.515 \\
ESTA & C2 & 0.323 & 0.731 & 0.535 \\
\hline ADMI 4007 & C3 & 0.209 & 0.391 & 0.153 \\
GEOP 4315 & C3 & 0.389 & 0.735 & 0.540 \\
FINA 3106 & C3 & 0.417 & 0.781 & 0.610 \\
FINA 3107 & C3 & 0.418 & 0.734 & 0.538 \\
\hline PRGR & C4 & 0.829 & 0.933 & 0.871 \\
Tiempo & C4 & 0.374 & 0.605 & 0.366 \\
\hline
\end{tabular}

Fuente: Elaboración propia.

Para medir la consistencia interna de cada constructo, se determinó el alpha de Cronbach y una mejor medida de consistencia interna basada en los loadings llamada rho de Dillon y Goldstein (DG. rho); en todos los casos son mayores de 0.7; además, los primeros autovalores (eigen) de la matriz de correlaciones de las variables asociadas al constructo son mayores de 1, tal como muestra la Tabla 5; y ratifican la consistencia interna en cada constructo.

\section{Tabla 5}

\begin{tabular}{lccccc}
\multicolumn{6}{l}{ Medidas de consistencia interna de cada constructo } \\
\hline Constructo & $\begin{array}{c}\text { Número de } \\
\text { variables }\end{array}$ & Cronbach & DG.rho & Eigen 1 & Eigen 2 \\
\hline C1 & 3 & 0.839 & 0.904 & 2.275 & 0.494 \\
C2 & 5 & 0.748 & 0.832 & 2.490 & 0.850 \\
C3 & 3 & 0.648 & 0.810 & 1.762 & 0.675 \\
C4 & 2 & 0.436 & 0.780 & 1.279 & 0.721 \\
\hline
\end{tabular}

Fuente: Elaboración propia. 
La Tabla 6 muestra los coeficientes positivos del sistema de relaciones lineales entre constructos representados en el modelo propuesto de la Figura 2, donde se muestran además las variables asociadas a cada constructo.

\section{Tabla 6}

Coeficientes significativos entre los constructos

\begin{tabular}{lcccc}
\hline $\begin{array}{l}\text { Relación de } \\
\text { Constructos }\end{array}$ & Coeficiente & Error estándar & Tvalue & Pvalue \\
\hline$C 1 \rightarrow C 2$ & 0.3533 & 0.0662 & 5.3402 & 0.0000 \\
$C 2 \rightarrow C 3$ & 0.6482 & 0.0538 & 12.0396 & 0.0000 \\
$C 2 \rightarrow C 4$ & 0.5638 & 0.0489 & 11.5373 & 0.0000 \\
$C 3 \rightarrow C 4$ & 0.3694 & 0.0489 & 7.5598 & 0.0000 \\
\hline
\end{tabular}

Fuente: Elaboración propia.

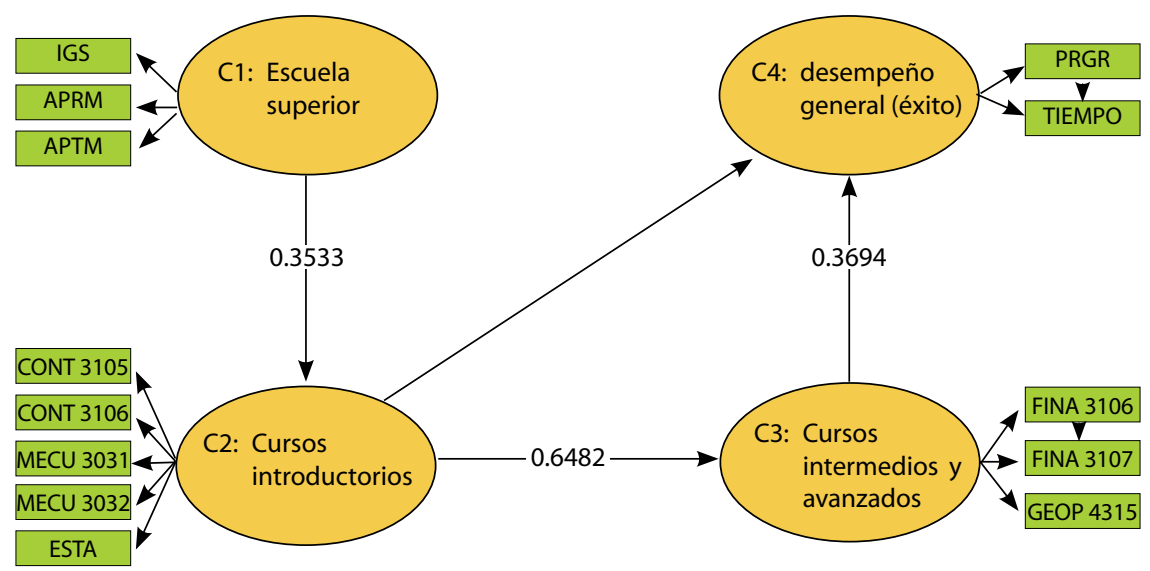

Figura 2. Relación significativa entre los constructos.

Fuente: Elaboración propia.

La prueba de coeficientes resultó altamente significativa en los cuatro casos; por lo tanto, se concluye que las cuatro hipótesis formuladas fueron validadas, es decir: 
1. El desempeño en la escuela superior influye positivamente en el desempeño en los cursos introductorios universitarios.

2. El desempeño en los cursos introductorios universitarios influye positivamente en el desempeño en los cursos intermedios y avanzados universitarios.

3. El desempeño en los cursos introductorios universitarios influye positivamente en el desempeño general (éxito).

4. El desempeño en los cursos intermedios y avanzados universitarios influye positivamente en el desempeño general (éxito).

\section{RL}

Para el análisis de los datos bajo estudio, mediante regresión logística, se definió una nueva variable llamada éxito. La nueva variable se refiere al éxito académico del estudiante y se definió como aquel alumno que culminó su bachillerato en cinco años o menos y obtuvo un promedio de graduación de tres puntos o más. De esta manera se encontraron 102 estudiantes de éxito académico, de un total de 202 estudiantes. Además, se promediaron las notas de los cursos que se ofrecen en dos partes, como son los cursos de contabilidad CONT 3105 y CONT 3106, los cursos de métodos cuantitativos MECU 3031 y MECU 3032, y los cursos de finanzas FINA 3106 y FINA 3107. En este caso, los promedios obtenidos definen nuevas variables; estas son: índice de los cursos de contabilidad (Icont), índice de los cursos de métodos cuantitativos (Imecu) e índice de los cursos de finanzas (Ifina).

El modelo de regresión logística propuesto establece que el éxito académico de un estudiante de la FAE depende de la influencia de las siguientes variables: género del estudiante, tipo de escuela, IGS, Icont, Imecu, Ifina, índice de los cursos de estadística (Iesta), índice del curso Gerencia Estratégica (Iadmi) e índice del curso Gerencia de Operaciones (Igeop).

La Tabla 7 muestra la evaluación del modelo de regresión logística propuesto. Se observa que cuatro variables no son significativas (n.s.); estas son: género del estudiante, Icont, Iadmi e Igeop; por lo tanto, hay cinco variables significativas que sí influyen en el 
éxito académico del estudiante: tipo de escuela, IGS, Imecu, Ifina e Iesta.

Tabla 7

Resultados del modelo de regresión logística propuesto

\begin{tabular}{lccccc}
\hline & Coeficiente & Error estándar & Zvalue & pvalue & \\
\cline { 2 - 6 } Intercept & -16.116 & 3.1659 & -5.09 & $<0.0001$ & \\
Género & 0.4252 & 0.3993 & 1.06 & 0.2869 & (n.s.) \\
Escuela & 1.7836 & 0.4408 & 4.05 & $<0.0001$ & \\
IGS & 0.0221 & 0.0089 & 2.48 & 0.0131 & \\
Imecu & 0.6124 & 0.2988 & 2.05 & 0.0404 & \\
Icont & -0.0481 & 0.3074 & -0.16 & 0.8757 & (n.s.) \\
Ifina & 1.1964 & 0.4101 & 2.92 & 0.0035 & \\
Iesta & 0.7192 & 0.2702 & 2.66 & 0.0078 & \\
Iadmi & 0.2018 & 0.2742 & 0.74 & 0.4617 & (n.s.) \\
Igeop & 0.0371 & 0.2478 & 0.15 & 0.8810 & (n.s.) \\
\hline
\end{tabular}

Fuente: Elaboración propia.

Se construyó un modelo de regresión logística para el éxito académico considerando las variables que resultaron significativas en el modelo que se ilustra en la Tabla 7. Se calcularon los OR y un intervalo del $95 \%$ de confianza para los mismos. Los resultados se muestran en la Tabla 8.

Tabla 8

Cálculo del OR de las variables significativas

\begin{tabular}{lccc}
\hline & OR & \multicolumn{2}{c}{ Intervalo de confianza $(95 \%)$} \\
\cline { 2 - 4 } Escuela & 5.802 & Inferior & Superior \\
\cline { 2 - 4 } IGS & 1.022 & 2.56 & 14.12 \\
Imecu & 1.895 & 1.01 & 1.04 \\
Ifina & 3.185 & 1.09 & 3.38 \\
Iesta & 2.139 & 1.55 & 7.07 \\
\hline
\end{tabular}

Fuente: Elaboración propia. 
De los resultados que muestra la Tabla 8 se puede concluir lo siguiente:

1. $O R_{\text {Escuela }}=5.802$; si se comparan los estudiantes que provienen de escuelas públicas y privadas, la posibilidad de encontrar un estudiante de éxito académico entre los que provienen de escuela privada es casi seis veces la posibilidad de encontrarlo entre los que provienen de escuela pública.

2. $O R_{I G S}=1.022$; si se comparan dos grupos de estudiantes diferenciados por 50 puntos de IGS; la posibilidad de encontrar un estudiante de éxito en el grupo de estudiantes con IGS mayor será casi tres veces $(1.02250=2.97)$ la posibilidad de encontrarlo entre los estudiantes de IGS menor; es decir, a mayor IGS, mayor la posibilidad de encontrar un estudiante de éxito.

3. $O R_{\text {Imecu }}=1.895$; si se comparan dos grupos de estudiantes diferenciados por un punto, en la escala de cero a cuatro, en la nota de los cursos de métodos cuantitativos, la posibilidad de encontrar un estudiante de éxito académico en el grupo de mayor nota será casi dos veces la posibilidad de encontrarlo entre los estudiantes con menor nota.

4. OR $_{\text {Ifina }}=3.185$; si se comparan dos grupos de estudiantes diferenciados por un punto, en la escala de cero a cuatro, en la nota de los cursos finanzas, la posibilidad de encontrar un estudiante de éxito académico en el grupo de mayor nota será algo más de tres veces la posibilidad de encontrarlo entre los estudiantes con menor nota.

5. $O R_{\text {Iesta }}=2.139$; si se comparan dos grupos de estudiantes diferenciados por un punto en la nota de los cursos de estadística, en la escala de cero a cuatro, la posibilidad de encontrar un estudiante de éxito académico en el grupo de mayor nota será el doble de la posibilidad de encontrarlo entre los estudiantes con menor nota. 


\section{Conclusiones}

A la luz de los resultados obtenidos en la investigación, se concluye lo siguiente:

1. En el análisis de datos mediante PLSPM, se observa que el índice de notas del curso ADMI 4007 fue excluido del modelo propuesto, ya que solo aporta el 15.3\% (y debe ser más del $45 \%$ ) de su variabilidad al constructo C3: desempeño en los cursos intermedios y avanzados; por lo tanto, tampoco influye en el constructo C4: desempeño general del estudiante.

2. El desempeño en la escuela superior (definido como la puntuación en el IGS, el aprovechamiento y la aptitud matemática) influye positivamente en el desempeño en los cursos introductorios: CONT 3105 y 3106, MECU 3031 y 3032, y ESTA 3041 y 3042 (o su equivalente ESTA 3045).

3. El desempeño en los cursos universitarios introductorios influye positivamente en el desempeño de los cursos intermedios y avanzados universitarios; es decir: FINA 3107 y 3106 y GEOP 4315.

4. El desempeño en los cursos universitarios introductorios, intermedios y avanzados influye positivamente en el desempeño general (promedio de graduación y tiempo de estudios).

5. En el análisis de datos mediante RL, se observa que estas cuatro variables no fueron significativas: género del estudiante e índice de notas de los cursos de contabilidad, gerencia estratégica y gerencia de operaciones. Hay cinco variables significativas que sí influyen en el éxito académico del estudiante; estas son: tipo de escuela, IGS y los índices de notas de los cursos de métodos cuantitativos, finanzas y estadística.

6. Se encontró que hay diferencias significativas en el tipo de escuela de la que proviene el estudiante; específicamente, 
se halló que la posibilidad de encontrar un estudiante de éxito académico entre los que provienen de escuela privada es casi seis veces la posibilidad de encontrarlo entre los que provienen de escuela pública.

7. Se encontró que si se comparan dos grupos de estudiantes diferenciados por 50 puntos de IGS, la posibilidad de encontrar un estudiante de éxito en el grupo de estudiantes con IGS mayor será casi tres veces la posibilidad de encontrarlo entre los estudiantes de IGS menor; es decir, a mayor IGS, mayor la posibilidad de encontrar un estudiante de éxito.

8. Al comparar el índice de los cursos de métodos cuantitativos en grupos diferenciados por un punto, se encontró que la posibilidad de hallar un estudiante de éxito académico en el grupo de mayor nota es casi dos veces la posibilidad de encontrarlo entre los estudiantes con menor nota.

9. Al comparar el índice de los cursos de finanzas en dos grupos de estudiantes diferenciados por un punto, se encontró que la posibilidad de hallar un estudiante de éxito académico en el grupo de mayor nota es algo más de tres veces la posibilidad de encontrarlo entre los estudiantes con menor nota.

10. Al comparar el índice de los cursos de estadística en dos grupos de estudiantes diferenciados por un punto, se encontró que la posibilidad de hallar un estudiante de éxito académico en el grupo de mayor nota es el doble de la posibilidad de encontrarlo entre los estudiantes con menor nota.

\section{Recomendaciones}

Los hallazgos de la investigación apuntan a las siguientes recomendaciones:

1. Motivar a los estudiantes a tomar los cursos en el orden establecido, según las secuencias curriculares de sus programas 
académicos, para que tengan una mayor posibilidad de tener éxito en la culminación de sus estudios universitarios. Las instituciones educativas deben tener un mayor acercamiento a sus estudiantes proveyendo orientación y asesoría académica durante su formación profesional, además de implantar actividades que los ayuden a determinar sus metas profesionales (Goncalves y Trunk, 2014).

2. Las universidades deben proveer programas de tutorías y laboratorios a los estudiantes que lo necesiten, ya que se ha comprobado que estos tienen una influencia positiva en la retención y el promedio general de los alumnos (Croft, Harrison, \& Robinson, 2009; Laskey \& Hetzel, 2011; Velázquez Rosado, Villafañe Cepeda, \& Vega Vilca, 2015).

3. Se deben estudiar otras variables que inciden en el éxito de los estudiantes en la culminación de sus estudios universitarios, tales como: sicológicas, sociales y económicas (Coronado Ramírez, Sandoval Bravo, \& Torres Mata, 2012). También se deben investigar las estrategias de aprendizaje de los estudiantes universitarios y su rendimiento académico (Martín, García, Torbay, \& Rodríguez, 2008); esto es así, pues se ha encontrado que los estudiantes universitarios de éxito son aquellos que utilizan estrategias motivacionales, autorregulan su estudio y revisan todo el proceso que realizan. 


\section{Referencias}

Arias Ortiz, E., \& Dehon, C. (2013). Roads to success in the Belgian French Community's higher education system: Predictors of dropout and degree completion at the Université Libre de Bruxelles. Research in Higher Education, 54, 693-723. doi 10.1007/s11162-013-9290-y

Coronado Ramírez, S., Sandoval Bravo, S., \& Torres Mata, A. (2012). Diferencias de género, factores que inciden en el rendimiento matemático de licenciaturas económico administrativas. Sinéctica, Revista Electrónica de Educación, 39. Recuperado de http:/ / www.sinectica.iteso.mx/index.php?cur=39\&art=39_11

Croft, A. C., Harrison, M. C., \& Robinson, C. L. (2009). Recruitment and retention of students-an integrated and holistic vision of mathematics support. International Journal of Mathematical Education in Science and Technology, 40(1), 109-125. doi 10.1080/00207390802542395

DeNicco, J., Harrington, P, \& Fogg, N. (2015). Factors of one-year college retention in a public state college system. Research of Higher Education Journal, 27, 1-13.

Goncalves, S. A., \& Trunk, D. (2014). Obstacles to success for the nontraditional student in higher education. Psi Chi Journal of Psychological Research, 19 (4), 164-72.

Gordon, S. (2010, Oct). The states of mathematics education today: What happens in the math classroom. Journal of Economics and Finance, 34, 471-476.

Gupta, S., Harris, D. E., Carrier, N. M., \& Caron, P. (2006). Predictors of student success in entry-level undergraduate mathematics courses. College Student Journal, 40(1), 97-108.

Larrazolo, N., Backhoff, E., \& Tirado, F. (2013). Habilidades de razonamiento matemático de estudiantes de educación media superior en México. Revista Mexicana de Investigación Educativa, 18, 1137-1163.

Laskey, M. C., \& Hetzel, C. J. (2011). Investigating factors related to retention of at-risk college students. Learning Assistance Review, 16(1), 31-43. 
Lesik, S. A. (2007). Do developmental mathematics programs have a causal impact on student retention? An application of discrete-time survival and regression-discontinuity analysis. Research in Higher Education, 48, 583-608. doi: 10.1007/s11162006-9036-1

Martín E., García, L. A., Torbay, A., \& Rodríguez, T. (2008). Estrategias de aprendizaje y rendimiento académico en estudiantes universitarios. International Journal of Psychology and Psychological Therapy, 8, 401-412.

Matos Díaz, H. (2008). Relaciones entre admisiones, calidad de los estudiantes, aprovechamiento académico e "inflación de calificaciones": Evidencia de la Universidad de Puerto Rico en Bayamón. Revista de Ciencias Sociales, 18, 78-103.

Parker, M. (2005). Placement, retention, and success: A longitudinal study of mathematics and retention. The Journal of General Education, 54(1), 22-40.

Sánchez, G. (2013). PLS path modeling with r. Berkeley, CA: Trowchez Editions. Recuperado de http://www.gastonsanchez. com/PLS_Path_Modeling_with_R.pdf

Vázquez Calle, F., \& Cabrera Pérez, J. A. (2004). Algunos determinantes del éxito en completar el bachillerato: Estudio longitudinal de seis años. Cayey, PR: Universidad de Puerto Rico en Cayey. Recuperado de http://portalwww.cayey.upr.edu/main/sites/ default/files/Algunos_determinantes_del_exito_en_completar_el_bachillerato_Estudio_Longitudinal_de_Seis_Anos.pdf

Vega Vilca, J. C., \& Agosto, F. (2010). Predicción del aprovechamiento académico: Estudio de los egresados de la FAE, UPRRP. Revista de Ciencias Sociales, 23, 130-143.

Velázquez Rosado, W., Villafañe Cepeda, W., \& Vega Vilca, J. C. (2015). El efecto del laboratorio en el aprovechamiento de los estudiantes del curso Métodos Cuantitativos para Administración de Empresas I. Fórum Empresarial, 20(1), 59-77. 